HEFAT2014

$10^{\text {th }}$ International Conference on Heat Transfer, Fluid Mechanics and Thermodynamics

14 - 16 July 2014

Orlando, Florida

\title{
EXPERIMENTAL INVESTIGATION OF THE EFFECT OF MULTIPLE SYNTHETIC JETS ON HEAT TRANSFER AND PRESSURE LOSS IN MINICHANNELS
}

\author{
David M. Sykes* \\ Mainstream Engineering Corporation \\ Rockledge, FL, USA \\ E-mail: dsykes@mainstream-engr.com
}

\author{
Andrew L. Carpenter, P.E. \\ Mainstream Engineering Corporation \\ Rockledge, FL, USA \\ E-mail: acarpenter@mainstream-engr.com
}

\section{ABSTRACT}

Synthetic jets can enhance minichannel thermal performance by adding net momentum flux into a fluid stream without adding mass flux. When incorporated into minichannels, these jets can impinge on the far wall, disrupt boundary layers, and enhance mixing within the channel, leading to high heat transfer coefficients. Many researchers have examined the effects of synthetic jets in microchannels and minichannels with single- and multi-phase flows using various fluids. This study aims to characterize the use of synthetic jets in minichannels that utilize an aqueous-glycol mixture with multiple jets along the length of a channel. Previous research has shown the use of synthetic jets can augment local heat transfer coefficients by two to three times the value of steady flow conditions using refrigerant as the working fluid. In this investigation, average heat transfer coefficients and pressure loss were measured experimentally for a range of synthetic jet operating conditions. Experiments were conducted with a minichannel array containing embedded thermocouples in order to directly measure local wall temperatures. Wall temperature measurements taken at various locations along the channel indicated that when synthetic jets were used the average heat transfer coefficient along the channel increased up to $81 \%$. The effects of momentum ratio, frequency, and bulk Graetz number on heat transfer and pressure loss is presented. This research shows that by using a synthetic jet array the pressure loss (i.e., pumping power) can be reduced by $78 \%$ while maintaining the heat transfer coefficient. Finally, an empirical correlation is presented that predicts the heat transfer enhancement due to synthetic jets over the range tested that has a mean absolute error (MAE) of only $5.5 \%$.

\section{INTRODUCTION}

Advances in electronics technology have led to reduced electronics' packaging size and higher power components.
This new generation of high heat flux devices requires the development of more effective thermal management systems to remove heat and to reduce peak operating temperatures. Integrating microchannels and minichannels into electronics has shown the ability to dissipate tremendous heat fluxes up to $800 \mathrm{~W} / \mathrm{cm}^{2}$ [1]; however, due to manufacturing difficulties and cost, this approach has rarely been implemented in commercial systems. Current practice is to physically attach minichannelbased heat sinks to the heat-generating electronics, limiting heat flux capability. As heat transfer demands for these minichannel-based heat sinks increase, further enhancements are required.

Synthetic jets are a net-zero mass flux jet created by alternating induction and expulsion of fluid through an orifice orthogonal to the bulk fluid stream. The difference in vortical structures during the induction and expulsion phases gives rise to a net added momentum flux away from the synthetic jet. This flux penetrates the bulk fluid stream and impinges the far wall of small channels. The impingement on the wall disrupts developing boundary layers and creates quasi-turbulence that enhances mixing and significantly increases heat transfer coefficients. Heat transfer coefficients are not only increased directly beneath the synthetic jet but are also increased both upstream [2] and downstream [3-5] of the synthetic jet as well, leading to a substantial heat transfer enhancement over a large region of the channel. When synthetic jets are placed at multiple locations along the channel, higher heat fluxes can be dissipated and peak operating temperatures of the targeted electronics device can be decreased. This benefit allows for pumped cooling loops to be operated at a lower flow rate with decreased pressure drop and still achieve equivalent heat transfer performance when compared to a conventional minichannel.

With ever-increasing demands for higher heat flux devices, practical applications for synthetic jet-enhanced microand minichannels can be foreseen. The goal of this paper is to demonstrate and measure the positive impact of multiple synthetic jets along a minichannel. Various operating 
conditions for an aqueous-glycol are investigated and increased average heat transfer coefficient is observed in comparison to a conventional channel. This has implications in providing enhanced cooling in high heat flux power electronics and other devices, especially on a platform that already use a glycol based cooling system.

We hypothesize that the heat transfer enhancement resultant from the incorporation of synthetic jets is a function of the momentum ratio, Graetz number, and frequency of synthetic jet operation, given constant fluid properties and geometry. The effects of each of these parameters are explored while keeping all other operational parameters constant. Using the general relationships between each of these parameters, an empirical correlation is developed assuming that the effects studied are mutually independent.

\section{NOMENCLATURE}

$\begin{array}{lll}A & {\left[\mathrm{~m}^{2}\right]} & \text { Area } \\ C_{p} & {[\mathrm{~kJ} / \mathrm{kgK}]} & \text { Specific Heat } \\ D & {[\mathrm{~m}]} & \text { Diameter } \\ D_{h} & {[\mathrm{~m}]} & \text { Hydraulic Diameter } \\ E u & {[-]} & \text { Euler Number } \\ f & {[\mathrm{~Hz}]} & \text { Synthetic Jet Frequency } \\ G r_{L} & {[-]} & \text { Graetz Number } \\ h & {\left[\mathrm{~W} / \mathrm{m}^{2} \mathrm{~K}\right]} & \text { Heat Transfer Coefficient } \\ k & {[\mathrm{~W} / \mathrm{mK}]} & \text { Thermal Conductivity } \\ L & {[\mathrm{~m}]} & \text { Length } \\ n & {[-]} & \text { Quantity } \\ N u & {[-]} & \text { Nusselt Number } \\ \dot{m} & {[\mathrm{~kg} / \mathrm{s}]} & \text { Mass Flow Rate } \\ \mu & {[\mathrm{Pa} \mathrm{s}]} & \text { Dynamic Viscosity } \\ P & {[\mathrm{~Pa}]} & \text { Pressure } \\ \rho & {\left[\mathrm{kg} / \mathrm{m}^{3}\right]} & \text { Fluid Density } \\ P r & {[-]} & \text { Prandtl Number } \\ Q & {[\mathrm{~W}]} & \text { Heat } \\ \bar{q} & {\left[\mathrm{~W} / \mathrm{m}^{2}\right]} & \text { Average Heat Flux } \\ R e & {[-]} & \text { Reynolds Number } \\ T & {[\mathrm{C}]} & \text { Temperature } \\ U & {[\mathrm{~m} / \mathrm{s}]} & \text { Velocity }\end{array}$

\begin{tabular}{lll}
\multicolumn{2}{l}{ Special Characters } \\
$B$ & {$[-]$} & Momentum Ratio \\
$z$ & {$[\mathrm{~m}]$} & Axial location, measured from inlet
\end{tabular}

Subscripts

$\begin{array}{ll}b & \text { Bulk } \\ c & \text { Cavity } \\ c h & \text { Characteristic } \\ f & \text { Flow } \\ h t & \text { Heat transfer } \\ i & \text { Inlet } \\ o & \text { Outlet } \\ p & \text { Piezo } \\ \mathrm{j} & \text { Jet } \\ \mathrm{w} & \text { Wall }\end{array}$

\section{EXPERIMENTAL SETUP}

A 50/50 aqueous propylene-glycol mixture (PGW) was used as the working fluid in a pumped loop cooling system. The schematic for the test loop is shown in Figure 1. A magnetically-coupled variable-speed pump was used to regulate the flow rate through the system. Downstream of the pump is a pressurizer/pulsation dampener that was used to set the system pressure, dampen any pulsations from the pump, and negate any thermal expansion effects from temperature changes in the fluid. A heater upstream of the synthetic jet-enhanced minichannel cooler (SJEMC) was used to control the inlet fluid temperature. A coriolis mass flow meter, calibrated RTDs, and a calibrated differential pressure sensor were used to measure the flow rate, inlet and outlet temperatures, and pressure drop through the SJEMC.

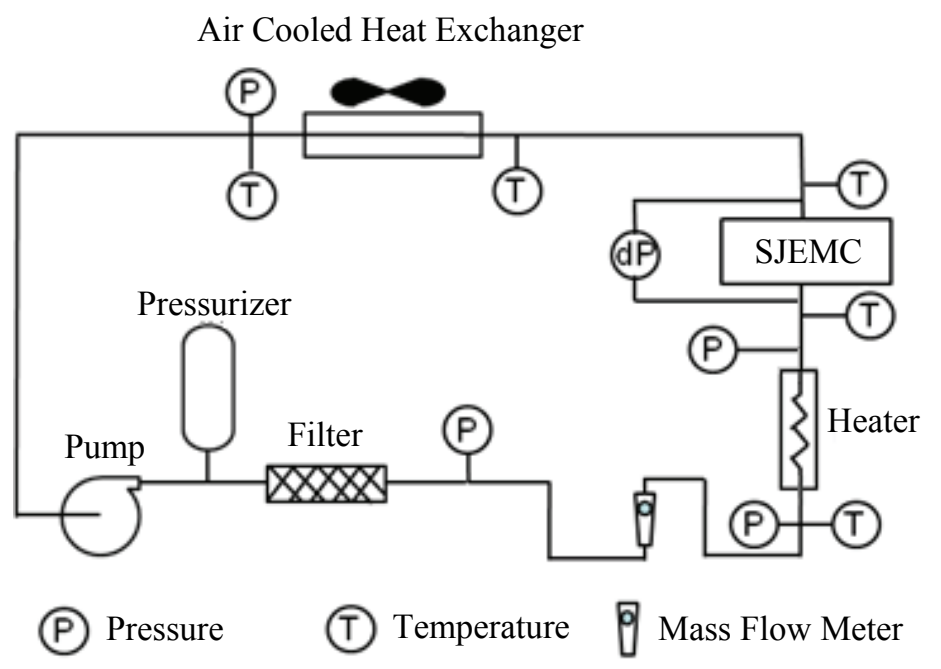

Figure 1: Test loop flow schematic

\section{Synthetic Jet-Enhanced Minichannel Cooler}

The geometry for the SJEMC is shown in Figure 2. The body of the cooler is split into two halves, top and bottom, with each half made of a low-thermal-conductivity phenolic/fiberglass composite material to reduce conductive heat losses. The top half contains the piston that is attached to a piezoelectric driving element, a fluid cavity, jet orifices, inlet and outlet plenums, and inlet/outlet connections (not shown). The reciprocating motion of the piezoelectrically driven piston within the cavity draws in and expels fluid through the orifices, producing the synthetic jets. The driving element is a directdrive piezoelectric actuator capable of a $750 \mu \mathrm{m}$ travel with a blocking force of $400 \mathrm{~N}$. The actuator is driven by an amplified variable frequency sine-wave function generator. The piezo stroke and frequency are measured via an external linear variable differential transformer (LVDT). The bottom half of the SJEMC body contains the minichannel insert and the mounting for the heater block, which is surrounded by a ceramic insulation. 


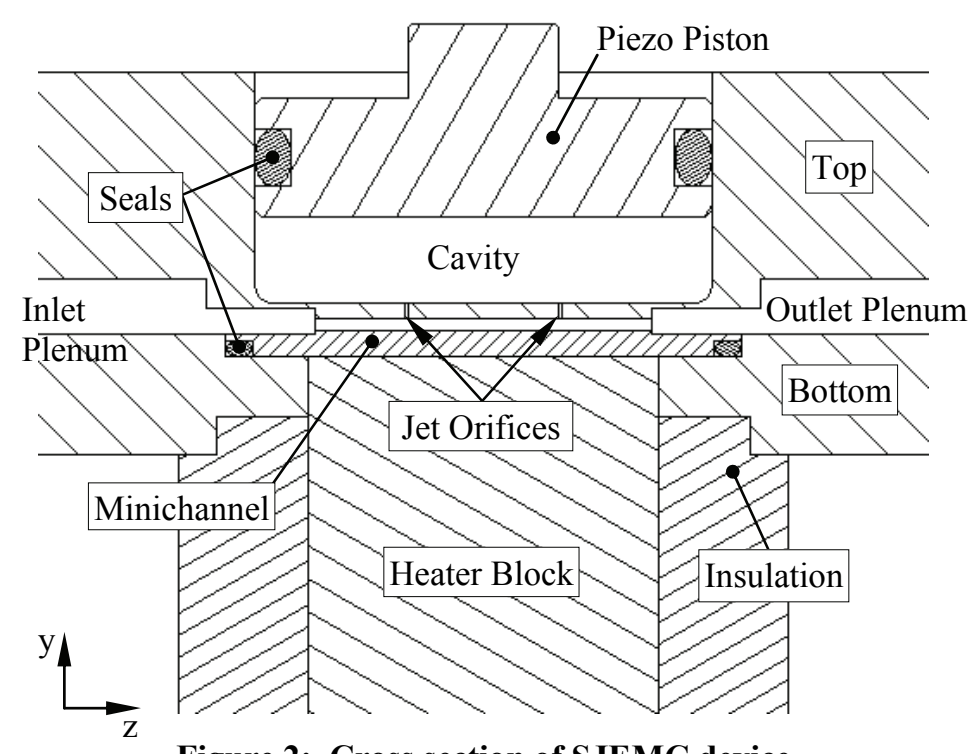

Figure 2: Cross section of SJEMC device

The minichannel insert is made from 6061 aluminum and contains 22 machined channels that measure $0.762 \mathrm{~mm}(\mathrm{w}) \mathrm{x}$ $1.0414 \mathrm{~mm}(\mathrm{~h}) \times 27.94 \mathrm{~mm}$ (l) and spaced by $0.508 \mathrm{~mm}$ fins. Each minichannel contains two synthetic jets that are located at $z / L=0.273$ and $z / L=0.727$, for a total of 44 synthetic jets. The orifice diameter and length for the jets are $0.254 \mathrm{~mm}$ and 1.27 $\mathrm{mm}$, respectively. Thermocouples were embedded into the minichannel to determine the channel bottom surface temperatures at discrete locations in order to calculate heat transfer coefficients. Eleven active thermocouples were soldered into the minichannel using solder with similar thermal conductivity to the aluminum to minimize distortion of the heat flux profile. The thermocouple junctions were located just $254 \mu \mathrm{m}$ from the channel surface to minimize heat spreading effects and conductive losses in wall temperature measurements as well as to provide quick response to transient events, such as activating the synthetic jet.
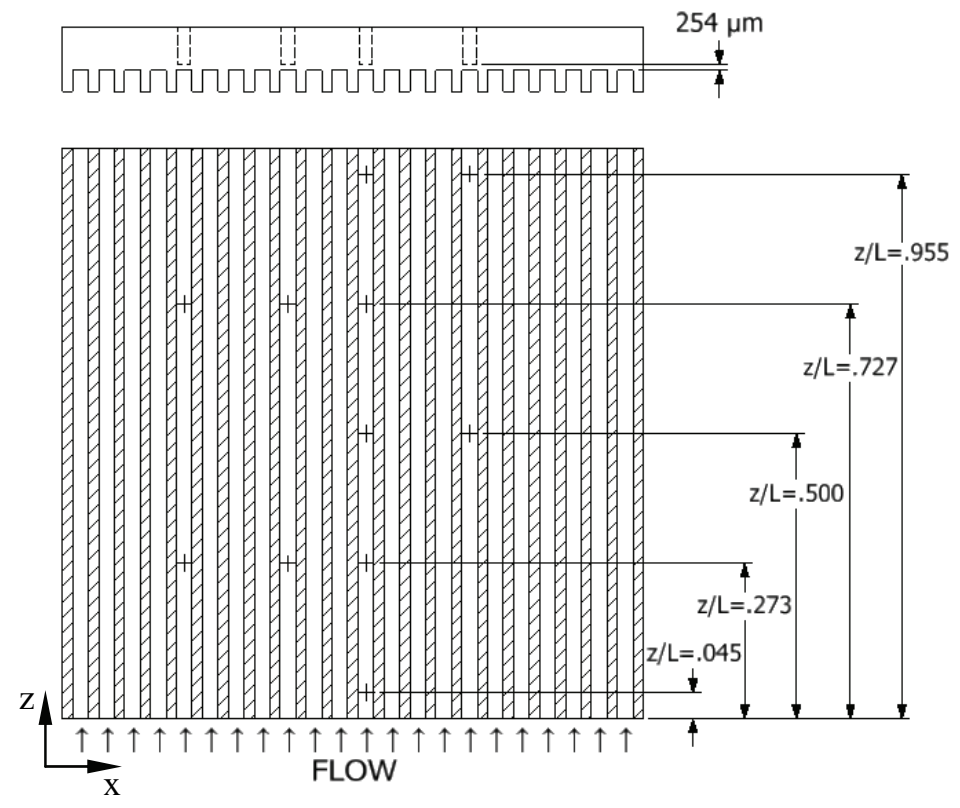

Figure 3: Minichannel thermocouple locations
The thermocouple locations are shown in Figure 3. Thermocouples were placed near the leading edge $(z / L=0.045)$, directly below the first row of synthetic jets $(z / L=0.273)$, at the midpoint of channel length $(z / L=0.500)$, directly below the second set of synthetic jets $(z / L=0.727)$, and downstream of the second set of synthetic jets $(z / L=0.955)$ along one of the center channels, and several span-wise channels, designated below by the center marks $(+)$ in Figure 3. The arrangement of thermocouples allowed for measurement of the effect of the synthetic jet directly below the jets and for investigation of any augmentation that occurs upstream and downstream of the jets. A thermal finite element analysis (FEA) was performed to confirm that the addition of the thermocouple and solder did not significantly alter the heat flux profile or temperature distributions on the minichannel.

\section{Experimental Procedure}

The experiments presented in the Results section were all performed using the following procedure:

1. Set the flowrate with the variable-speed pump.

2. Set the inlet temperature by adjusting the heater upstream of the SJEMC.

3. Set the system pressure using the pressurizer/pulsation dampener.

4. Apply the desired heat flux to the bottom of the minichannel insert.

5. Allow the inlet, outlet, and minichannel surface temperatures to achieve steady state (defined as a rate of change of less than $0.1^{\circ} \mathrm{C} / \mathrm{min}$ ).

6. Record the steady-state data for the desired 10 minutes. Data is averaged over this time period. This is the baseline $(0 \mathrm{~Hz})$ case.

7. Activate the synthetic jet at the desired frequency and amplitude to achieve the desired conditions.

8. Allow the inlet, outlet, and minichannel surface temperatures to achieve steady state (defined as a rate of change of less than $0.1{ }^{\circ} \mathrm{C} / \mathrm{min}$ ).

9. Record the steady-state data for 10 minutes. Data is averaged over this time period. This is the synthetic jet case for each test point.

10. Repeat steps 7-9 for each test condition.

For all tests, the inlet pressure to the SJEMC was held constant near $20 \mathrm{kPa}$, the inlet temperature was $333 \mathrm{~K}$, and the applied heat flux was held constant at $5 \mathrm{~W} / \mathrm{cm}^{2}$. The operation of the SJEMC was varied in several manners to study the augmentation effects. The hypothesis was that the heat transfer augmentation from operating the synthetic jet is a function of the momentum ratio, frequency of synthetic jet operation, and the bulk Reynolds number $\left(R e_{b}\right)$. The experiments performed sought to isolate these three effects by varying each while keeping all other parameters constant. Within the experiments, the parameter ranges for the bulk flow Reynolds number, synthetic jet frequency, and momentum ratio ranged from 56 to 183,0 to $75 \mathrm{~Hz}$, and 1.25 to 53.4 , respectively. 


\section{Data Analysis}

For every test condition, the heat transfer coefficient can be calculated for the synthetic jet on and the synthetic jet off cases. Based on comparison to uniform heat transfer correlations (discussed in Results), it was determined that there was not uniform heat transfer through the minichannel. Because of this non-uniform heat flux, the average heat transfer coefficient $\bar{h}$, was used to quantify thermal performance and was calculated by

$$
\bar{h}=\frac{\dot{\mathrm{m}} C_{p}\left(T_{o}-T_{i}\right)}{A_{h t}\left(\bar{T}_{w, \text { cond }}-T_{f, \text { ave }}\right)}
$$

where $A_{h t}$ is the heat transfer area of the minichannel, $\bar{T}_{w, \text { cond }}$ is the average wall temperature calculated based on the thermocouple temperature measurements and the conduction equation weighted by channel position, and $T_{f, a v e}$ is the average of the inlet and outlet fluid temperatures. Heat transfer is nondimensionalized by determining the average Nusselt number for the minichannel, which uses the average heat transfer coefficient calculated in equation 1 , and was defined as

$$
\overline{N u}=\frac{\bar{h} D_{h}}{k}
$$

where $D_{h}$ is the hydraulic diameter of a single minichannel $(0.880 \mathrm{~mm})$, and $k$ is the thermal conductivity of the fluid.

Flow effects were examined based on the Graetz number, which uses Reynolds number for these experiments as defined by

$$
R e=\frac{\rho U_{b} D_{h}}{\mu}
$$

where $\rho$ is the fluid density, $U_{b}$ is the bulk velocity in the channel, and $\mu$ is the dynamic viscosity of the fluid. The bulk velocity was derived from the measured mass flow rate by

$$
U_{b}=\frac{\dot{m}}{n \rho A_{f}}
$$

where $\dot{m}$ is the total mass flow measured by the coriolis flow meter, $n$ is the number of minichannels, $\rho$ is the fluid density, and $A_{f}$ is the flow area of each minichannel.

In addition to the flow and heat transfer calculations, the non-dimensional pressure loss Euler number, $E u$, was calculated to examine the effect that the synthetic jet had on pressure drop in the minichannel. The Euler number was defined as

$$
E u=\frac{\Delta P}{\rho U_{b}^{2}}
$$

where $\Delta P$ is the measured pressure drop across the channels, $\rho$ is the fluid density, and $U_{b}$ is the bulk velocity through the minichannel.

An important factor in investigating the effect of synthetic jets within minichannels was the strength of the synthetic jet. The strength of the synthetic jet and the degree of impingement on the minichannel surface is characterized by the relative magnitudes of the synthetic jet momentum and the bulk flow momentum. The previously characteristic velocity, $U_{c h}$, of the synthetic jet, is defined by Travnicek [6] as

$$
U_{c h}=\frac{D_{c}^{2}}{n_{j} D_{j}^{2}} L_{P} f
$$

where $D_{c}$ is the cavity diameter, $n_{j}$ is the number of synthetic jet orifices, $D_{j}$ is the orifice diameter, $L_{p}$ is the stroke length of the piezoelectric piston, and $f$ is the synthetic jet operating frequency. This characteristic velocity is the time averaged velocity through the orifices. The momentum ratio determines the degree of jet penetration into a crossflow, and thus, the degree of impingement on the heated minichannel surface. The degree of impingement is directly related to boundary layer disruption and stagnation heat transfer. The heat transfer augmentation from the synthetic jet is thus correlated to the momentum ratio, $B$, defined as

$$
B=\frac{U_{c h}^{2}}{U_{b}^{2}}
$$

To quantify the enhancement in thermal performance due to the synthetic jet, an average Nusselt number ratio is presented and was defined as

$$
\frac{\overline{N u}}{\overline{N u}_{O}}=\frac{\overline{N u}_{O N}}{\overline{N u}_{O F F}}
$$

where $\overline{N u}_{O N}$ is the average Nusselt number when the synthetic jet is operating, and $\overline{N u}_{O F F}$ is the average Nusselt number when the synthetic jet is not operating. Similarly, an Euler number ratio is presented to characterize the pressure loss and was defined as

$$
\frac{E u}{E u_{o}}=\frac{E u_{O N}}{E u_{O F F}}
$$

where $E u_{O N}$ is the Euler number when the synthetic jet is operating, and $E u_{O F F}$ is the Euler number for the minichannel when the synthetic jet is not operating.

Finally, the low $z / D_{h}$ ratio of the thermocouple locations indicated that the flow would not be thermally fully developed. Thermal entrance effects are captured by the Graetz number, which was defined as

$$
G z_{L}=\frac{R e P r}{L / D_{h}}
$$

where $L$ is the length of the minichannel, $D_{h}$ is the hydraulic diameter of the minichannel, $R e$ is the Reynolds number, and $\operatorname{Pr}$ is the Prandtl number.

\section{RESULTS}

Equations 1-10 were used to calculate all results presented here. Fluid properties, such as $\rho, C_{p}, k, \mu$, and $P r$, were calculated for each test condition based on the average fluid temperature in the channel based on aqueous mixture correlations [7].

\section{Heat Transfer Comparison across Different Channels and Modes of Operation}

Temperature measurements were taken in different minichannels at the same z-location to determine if any heat transfer nonuniformities caused by maldistribution of flow 
could be found. Figure 4 shows a typical variation of temperature measurements from three different channels at the axial location $z / L=0.727$ over a span of momentum ratios. The momentum ratio is very sensitive to bulk flow velocity and, as discussed below, the momentum ratio has a large effect on heat transfer coefficient. Thus, maldistribution in bulk flow would cause a significant surface temperature variation. The variation of the measurements between each channel was lower than the measurement error of the thermocouples and also showed a random relationship with respect to momentum ratio. Therefore, the assumption was that the flow distribution was good and that each of the channels experienced similar heat transfer characteristics.

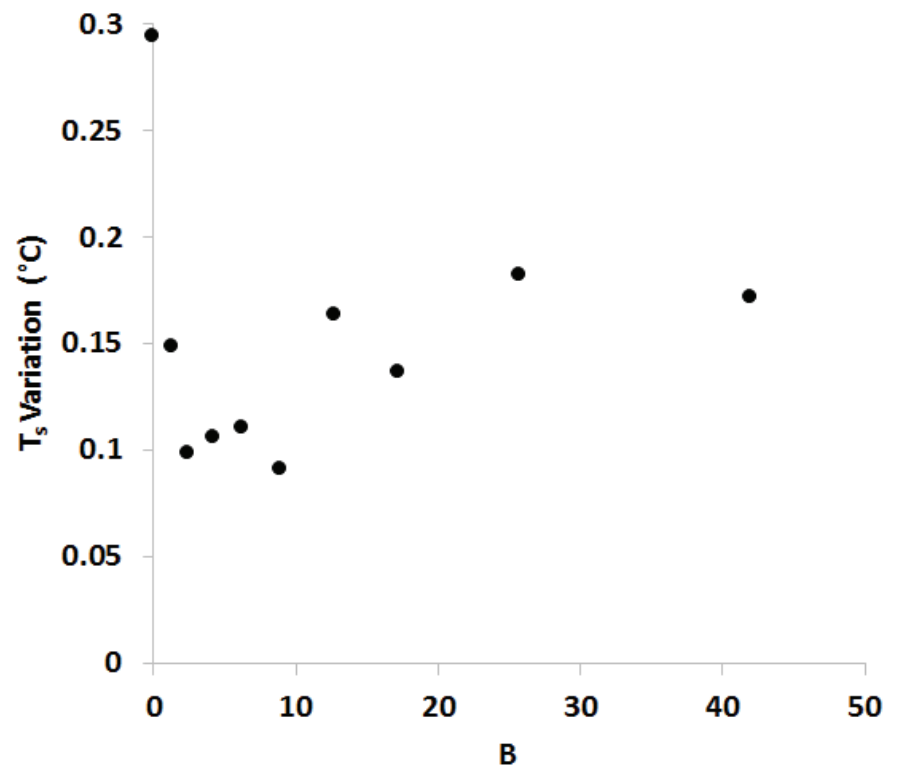

Figure 4: Surface temperature variation at $z / L=0.727$ during a momentum ratio sweep

Figure 5 shows the temperature difference between the surface of the minichannel and the local bulk average fluid temperature as a function of the streamwise location and operation of the synthetic jet. In this case, the local bulk average fluid temperature was calculated assuming uniform heat flux at the wall. For the synthetic jet off case (i.e. conventional minichannel flow), the surface temperature profile did not match classical theory, which predicts an increasing temperature difference approaching an asymptote at the thermally fully-developed condition. The measured temperature profile showed a negative slope in temperature difference for $z / L$ larger than 0.6. This indicates that the heat flux distribution was not uniform.

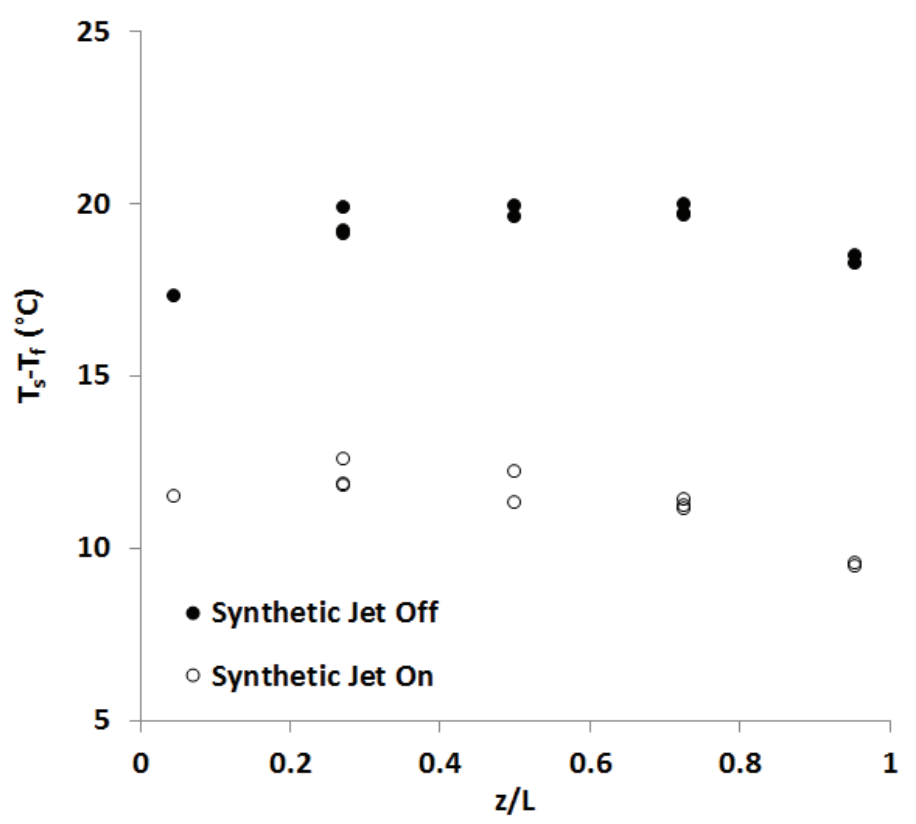

Figure 5: Temperature difference between the surface and the fluid as a function of streamwise location

To further this assertion, the wall temperature was calculated as a function of the normalized length for the channel geometry and was based on the assumption of uniform heat flux using a well-established theoretical correlation from Lee and Garimella [8]. Figure 6 shows this theoretical wall temperature calculation and the measured data. The shape of the curves does not match with the point near the leading edge showing substantially higher than predicted temperatures, and the trailing edge showing substantially lower than predicted temperatures.

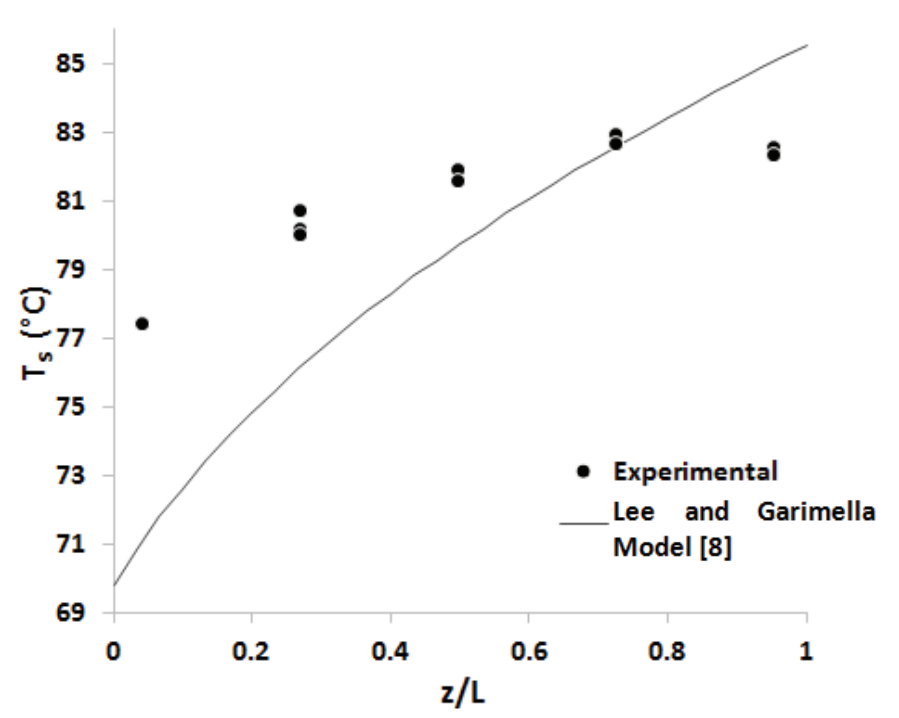

Figure 6: Wall temperature as a function of normalized length for a selected condition 
The discrepancy between the theoretical calculation and the measured results prompted a higher fidelity model of the heat transfer through the minichannel. A thermal finite element analysis (FEA) was performed with the same boundary conditions as one of the experimental data points. Figure 7 shows a heat flux contour of the minichannel array calculated by the analysis. The results show a large change in heat flux from the inlet of the minichannels (bottom) to the outlet of the minichannels (top). The belief is that the varying heat transfer coefficients, conduction pathways within the minichannel, and varying fin efficiencies along the minichannel length all contributed to the non-uniform heat flux. Because neither the uniform heat flux nor uniform surface temperature boundary conditions existed in the minichannel, a measurement of local heat flux would be required to calculate local Nusselt numbers.

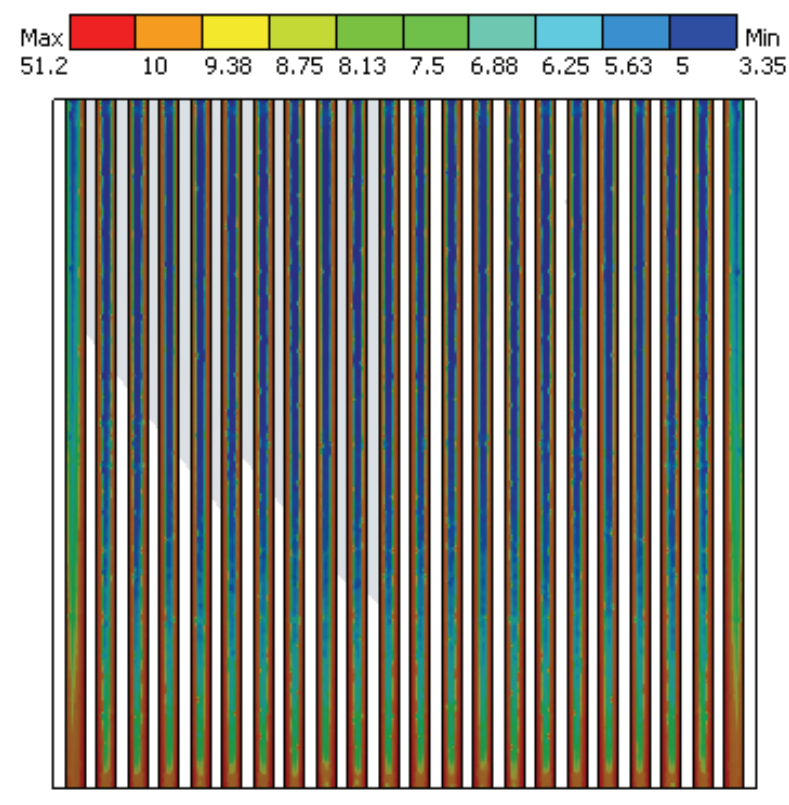

Figure 7: FEA results showing heat flux $\left(w / \mathrm{cm}^{2}\right)$ distribution along the channel length

Figure 5 also shows the temperature difference between surface and local bulk average fluid temperature as a function of the streamwise location while the synthetic jet is operating. While the temperature difference reduced at all streamwise locations, indicating enhanced heat transfer, the temperature profile was also altered. The negative slope at $z / L$ greater than 0.6 is larger for the synthetic jet on case and the leading edge experienced a lower decrease in temperature difference than downstream locations. The altered shape of the profile also indicates that the heat flux distribution along the channel changed from the synthetic jet off case shown in Figure 7. If the local heat flux were invariant with synthetic jet operation, the Nusselt number ratio between the synthetic jet on and off cases would simply be a ratio of wall-fluid temperature differences. However, because the heat flux distribution was altered when operating the synthetic jet to an unknown profile, it was not possible to calculate the local augmentation of the Nusselt number with the integrated thermocouples alone.
Therefore, to quantify the heat transfer enhancement due to the synthetic jets, the average Nusselt numbers are presented.

\section{Effect of Momentum Ratio}

Previously in the literature, it has been reported that the heat transfer ratio is a strong function of the momentum ratio [2]. However, these previous tests were conducted with a different fluid at transitional and turbulent Reynolds numbers. In the current investigation, the Reynolds numbers were restricted to the laminar regime. To examine the effect of momentum ratio in this flow regime, the Reynolds number, Prandtl number, heat flux, and frequency were all held constant while varying the amplitude of the piston oscillation (the stroke, $L_{p}$ ) to modulate the momentum ratio. Specific conditions for the experiment were:

$$
\begin{array}{ll}
\text { - } & \mathrm{T}_{\mathrm{i}}=333 \mathrm{~K} \\
\text { - } & \mathrm{f}=30 \mathrm{~Hz} \\
\text { - } & \mathrm{Re}=183 \\
\text { - } & \mathrm{Pr}=12.9 \\
\text { - } & \mathrm{q} "=5.03 \mathrm{~W} / \mathrm{cm}^{2}
\end{array}
$$

Figure 8 shows a graph of the heat transfer augmentation (i.e., ratio of Nusselt numbers for the synthetic jet on and off cases) as a function of momentum ratio for the synthetic jets. As shown previously, using synthetic jets increases heat transfer significantly. The heat transfer enhancement has a fractional power relationship with the momentum ratio. For this range of experiments the heat transfer coefficient increased between $27 \%$ and $71 \%$ depending on momentum ratio.

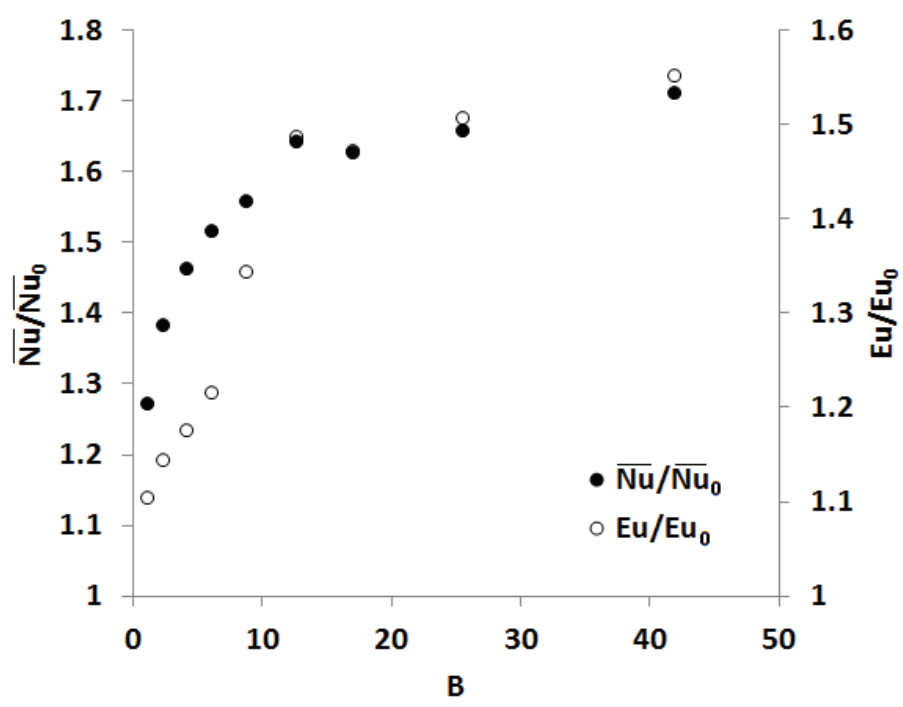

Figure 8: Heat transfer enhancement as a function of momentum ratio

Even though the heat transfer enhancement increases with momentum ratio, a thermal system designer must be cognizant of the pressure loss penalty incurred to achieve the heat transfer enhancement. Figure 8 also shows the pressure loss ratio (i.e. ratio of pressure loss for the synthetic jet on and off cases) as a function of the momentum ratio. Initially, as the 
momentum ratio increased, the heat transfer enhancement increased rapidly until $\mathrm{B}=12.7$. Further increase in the momentum ratio yielded a marginal increase in heat transfer performance. However, above $\mathrm{B}=6.2$ a large increase in pressure loss occurred. The belief is that this behavior was caused by the shift in dominant flow mechanism from disturbed channel flow to impingement flow. The shift in performance also implies the existence of an optimum momentum ratio to operate the synthetic jet array. In this case, the optimum momentum ratio that maximizes heat transfer enhancement while minimizing pressure loss penalty is 6.21 for the stated operating conditions. The heat transfer enhancement for this point was $51.5 \%$ while the pressure loss increase was only $21 \%$.

\section{Effect of Synthetic Jet Frequency}

The above data shows a strong effect of the momentum ratio on the heat transfer enhancement and pressure loss of the minichannel. In this experiment, the effect of synthetic jet frequency was examined. The Reynolds number, Prandtl number, heat flux, and momentum ratio were held constant at the values listed below, while the frequency and the amplitude of synthetic jet oscillation were varied.

- $\mathrm{T}_{\mathrm{i}}=333 \mathrm{~K}$

- $\mathrm{B}=35$

- $\operatorname{Re}=182$

- $\operatorname{Pr}=12.8$

- $\quad \mathrm{q} "=5.07 \mathrm{~W} / \mathrm{cm}^{2}$

Figure 9 shows a graph of the Nusselt number enhancement and Euler number ratio as a function of synthetic jet operating frequency.

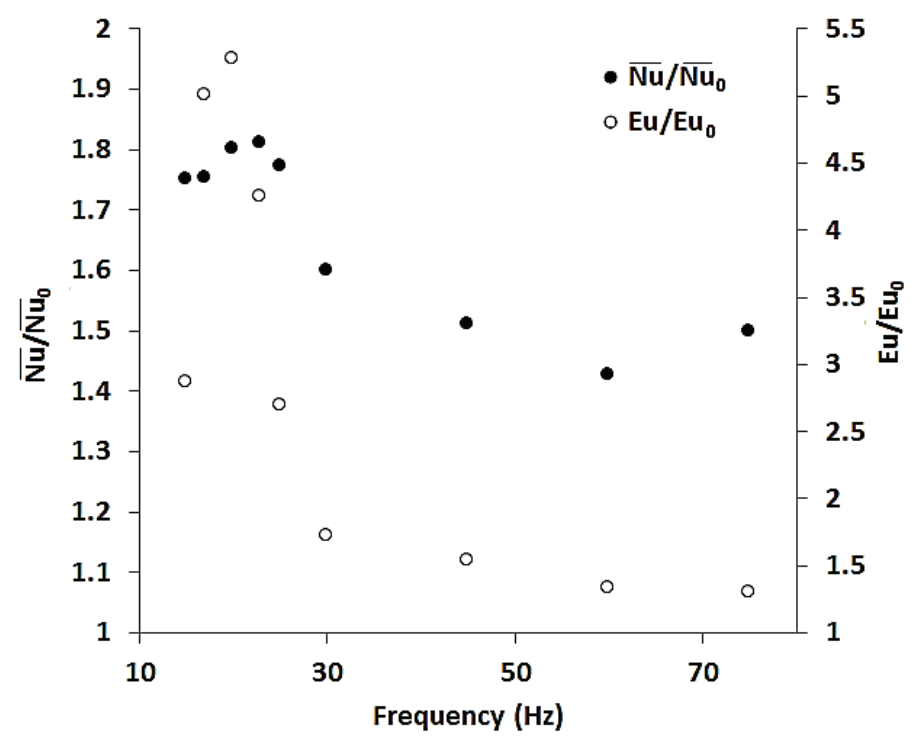

Figure 9: Heat transfer enhancement and pressure loss ratio as a function of synthetic jet operating frequency

A resonant peak was observed at approximately $20 \mathrm{~Hz}$. Around this frequency, the heat transfer enhancement and pressure loss across the minichannel increased dramatically. Unfortunately, the pressure loss ratio (maximum of 5.28) increased much more than the heat transfer enhancement (maximum of 1.80). Therefore, in practical systems avoiding the system resonances is advantageous unless the system can be precisely operated near but not at the resonant frequency. For instance, at $30 \mathrm{~Hz}$ the peak heat transfer enhancement was 1.60 , which was substantially higher than the enhancement at $45 \mathrm{~Hz}$. Also, the pressure loss ratio was only 1.71 , which was similar to the ratios seen at $45 \mathrm{~Hz}$ and higher operating frequencies.

\section{Effect of Graetz Number}

The average Nusselt number for the minichannel flow is a function of the Graetz number because it is still thermally developing. Therefore, the effect of varying the Graetz number by changing the Reynolds number was examined in this experiment. The Prandtl number, heat flux, momentum ratio, and synthetic jet operating frequency were held constant at the values listed below, while the Reynolds number and the amplitude of synthetic jet oscillation were varied.

$$
\begin{array}{ll}
\text { - } & \mathrm{T}_{\mathrm{i}}=333 \mathrm{~K} \\
\text { - } & \mathrm{B}=50 \\
\text { - } & \mathrm{f}=35 \mathrm{~Hz} \\
\text { - } & \mathrm{Pr}=12 \\
\text { - } & \mathrm{q}=5.05 \mathrm{~W} / \mathrm{cm}^{2}
\end{array}
$$

Figure 10 shows the Nusselt number and Euler number ratios as a function of the Graetz number.

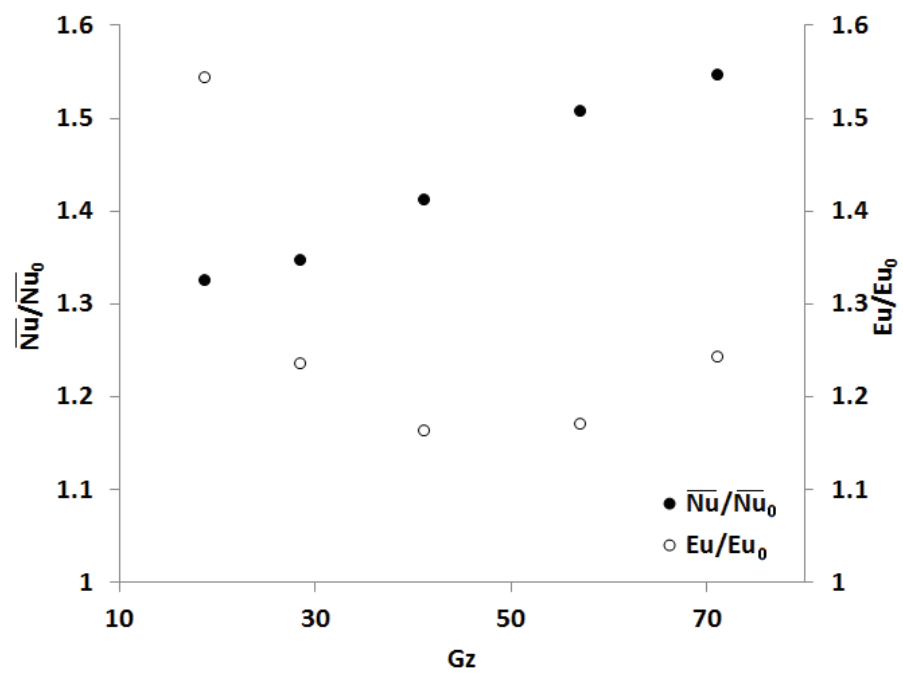

Figure 10: Heat transfer enhancement and pressure loss ratio as a function of the Graetz number

A more practical viewpoint of the above data is shown in Figure 11 where the average heat transfer coefficient is plotted against the pressure loss through the minichannel for both synthetic jet on and off cases. Both cases show a relatively linear profile over the pressure loss range, with the synthetic jet on case showing a $76 \%$ steeper slope. 


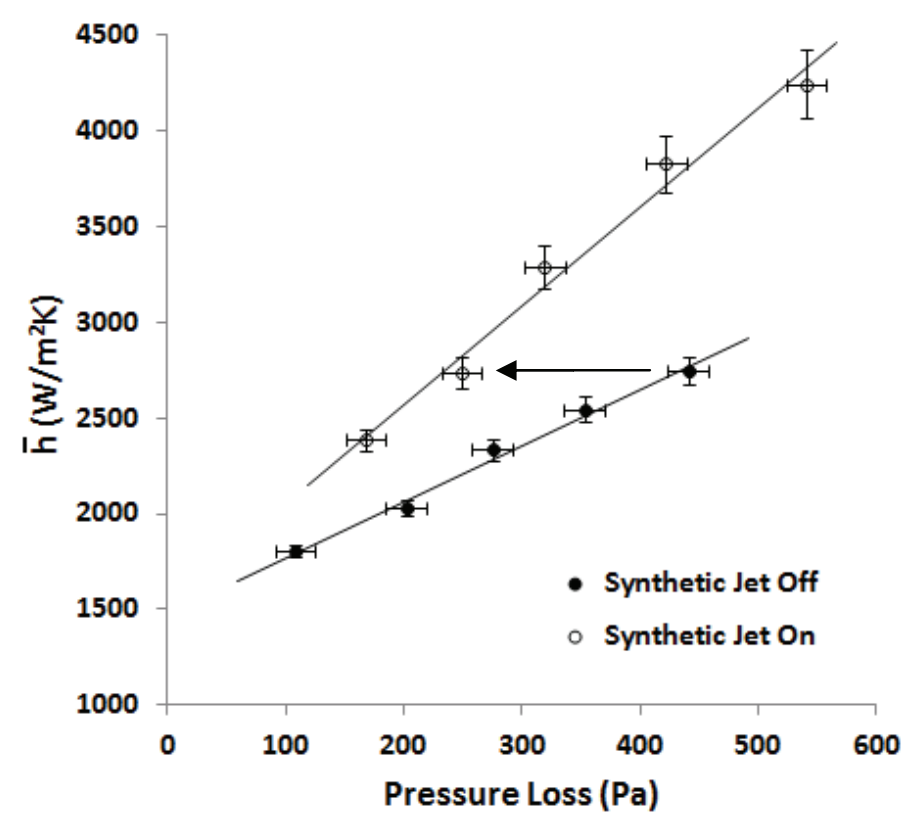

Figure 11: Average heat transfer coefficient as a function of synthetic jet operation and pressure loss

Effectively, the steeper slope indicates that using synthetic jets is more advantageous than simply increasing the flowrate through the minichannel. Additionally, the advantage of using the synthetic jets increases as desired heat transfer coefficients increases. For instance, assume that this minichannel was used to cool electronics and that the average heat transfer coefficient needed to be $2735 \mathrm{~W} / \mathrm{m}^{2} \mathrm{~K}$. A simple minichannel could be used at a flow rate of $4.65 \mathrm{~g} / \mathrm{s}$, and the incurred pressure loss would be $440 \mathrm{~Pa}$. Alternatively, if the minichannel with the synthetic jet was used, the flow rate could be reduced to $1.86 \mathrm{~g} / \mathrm{s}$ and the pressure loss incurred would be only $249 \mathrm{~Pa}$ (designated by the arrow in Figure 11). This represents a $77.4 \%$ reduction in pressure loss, and therefore, pumping power.

\section{ERROR ANALYSIS}

The error bars reported in Figure 11 were due to measurement uncertainties. Table 1 shows the transducer measurement uncertainties and the geometric uncertainty from machining and measurement variance. Error in fluid and material property data $\left(\rho, C_{p}, k, \mu\right.$, and $\left.\operatorname{Pr}\right)$ was assumed to be negligible across the uncertainty range of temperatures.

Table 1: Geometric and Transducer Uncertainty

\begin{tabular}{|l|c|c|}
\hline \multicolumn{1}{|c|}{ Variable } & Error ( \pm ) & Units \\
\hline Inlet Temperature, $T_{i}$ & $0.15+0.002^{*} T_{i}$ & ${ }^{\circ} \mathrm{C}$ \\
\hline Outlet Temperature, $T_{o}$ & $0.15+0.002^{*} T_{o}$ & ${ }^{\circ} \mathrm{C}$ \\
\hline Wall Temperature, $T w$ & 1.5 & ${ }^{\circ} \mathrm{C}$ \\
\hline Pressure Drop, $\Delta P$ & 17.24 & $\mathrm{~Pa}$ \\
\hline Mass Flow Rate, $\dot{m}$ & $1.225 \times 10^{-6}-4.67 \times 10^{-6}$ & $\mathrm{~kg} / \mathrm{s}$ \\
\hline Hydraulic Diameter, $D_{h}$ & $1.922 \times 10^{-5}$ & $\mathrm{~m}$ \\
\hline Heat Transfer Area, $A_{h t}$ & $3.491 \times 10^{-5}$ & $\mathrm{~m}^{2}$ \\
\hline
\end{tabular}

The individual measurement uncertainties propagate to the reported values according to the relation seen in Equation 11. Equation 11 shows the generic estimation of error in function $F$, assuming that $F=f\left(x_{1}, x_{2}, \ldots, x_{n}\right)$. This approach determines the probable error of the function by using the Pythagorean summation of the discrete uncertainties associated with the function. The contribution of the discrete uncertainties are weighted by multiplying the error in the variable of interest by the partial derivative of the function with respect to that variable.

$$
e_{F}=\sqrt{\left(e_{x_{1}} \frac{\partial F}{\partial x_{1}}\right)^{2}+\left(e_{x_{2}} \frac{\partial F}{\partial x_{2}}\right)^{2}+\cdots+\left(e_{x_{n}} \frac{\partial F}{\partial x_{n}}\right)^{2}}
$$

\section{CORRELATION DEVELOPMENT}

The findings in the Results section indicated that the average heat transfer enhancement due to synthetic jets is a function of the momentum ratio, the frequency of oscillation, and the Graetz number. The Graetz number effect appeared to be linear, the momentum ratio effect appeared to be a power relation, and the frequency effect appeared to have a harmonic relationship. If one assumes that these effects are independent, then the following functional form applies:

$$
\frac{\overline{N u}}{\overline{N u_{o}}}=1+\left(C_{1} G z+C_{2}\right) B^{C_{3}} e^{-C_{4} F^{2}}
$$

where $F$ is the difference between the synthetic jet frequency and the apparent resonance frequency, normalized by the resonance frequency. Using standard nonlinear regression software, the constants $\mathrm{C}_{1}, \mathrm{C}_{2}, \mathrm{C}_{3}$, and $\mathrm{C}_{4}$ were solved for using the entire data set. The resulting values for the constants were as follows:

$$
\begin{aligned}
& \mathrm{C}_{1}=.002391 \\
& \mathrm{C}_{2}=.1953 \\
& \mathrm{C}_{3}=.1902 \\
& \mathrm{C}_{4}=.09719
\end{aligned}
$$

Figure 12 shows the ability of the correlation to estimate the experimental measurements. The correlation predicts the behavior well, and the mean absolute error in heat transfer enhancement is only $5.5 \%$. This correlation is only valid for 50/50 PGW mixtures because even though this correlation fits the data well, there are other effects that are not included in the correlation. For instance, one can anticipate a strong effect of viscosity on the average heat transfer enhancement that is not appropriately captured in the correlation. Nonetheless, the correlation does show the correct behaviour and matches experimentally observed trends. 


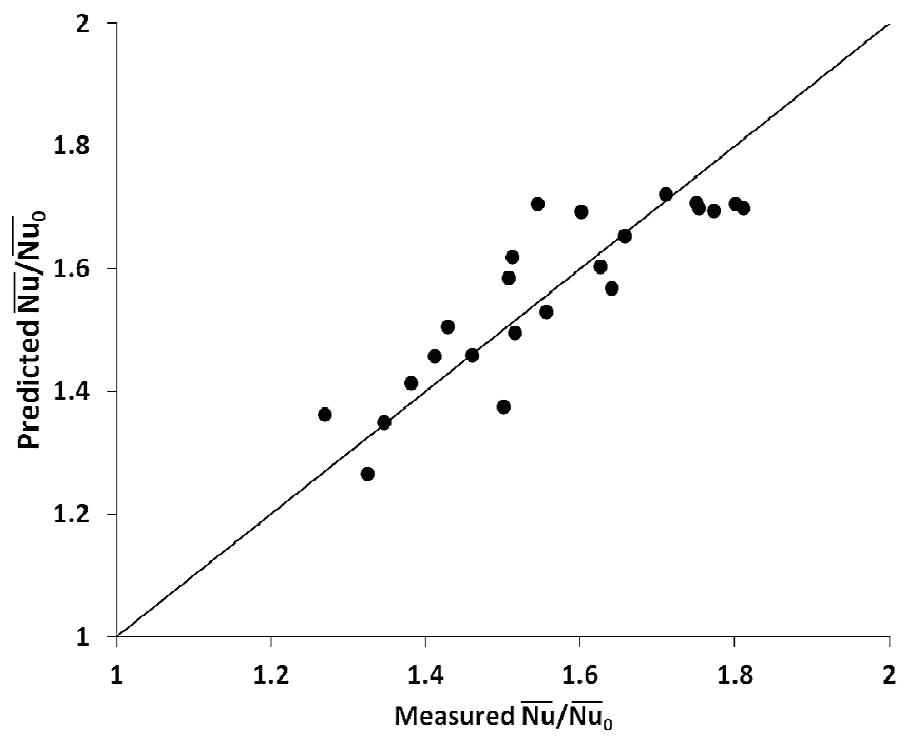

Figure 12: Correlation predicted heat transfer enhancement versus measured heat transfer enhancement

\section{CONCLUSIONS}

A series of experiments were performed to assess the heat transfer and pressure loss impacts of using synthetic jets on a minichannel array. Key conclusions are:

1. Heat transfer enhancements of 27 to $81 \%$ were measured when the synthetic jets were active for this range of experiments.

2. Heat transfer enhancement appears to be a linear function of Graetz number, power function of momentum ratio, and Gaussian function of the operating frequency relative to the apparent resonant frequency.

3. The slope of average heat transfer coefficient versus pressure loss is steeper when the synthetic jet is on. This implies that large reductions in incurred pressure loss are possible at high flow rates while maintaining desired heat transfer performance.

4. Pressure loss ratios follow similar trends as heat transfer enhancement for momentum ratio effects and frequency effects. However, the pressure loss magnitude is much higher than the heat transfer enhancement near the apparent resonant peak.

5. A correlation was developed based on the observed effects of frequency, momentum ratio, and Graetz number that has an MAE of only 5.5\%.

\section{ACKNOWLEDGEMENTS}

The authors acknowledge the technical support from Mrs. Mary Goryca of the U.S. Army TACOM.

The work presented here is a result from a SBIR contract awarded by U.S. Army TACOM (TARDEC) (Contract \#: W56HZV-09-C-0060)

\section{REFERENCES}

[1] Tuckerman, D. and Pease, R. "High Performance Heat Sinking for VLSI," IEEE Electron Device Letters, 1981, Vol. EDL-2, pp.126-129.

[2] Sykes, D., Carpenter, A., and Cole, G., "Experimental Investigation of the Effect of Synthetic Jets on Local Heat Transfer Coefficients in Minichannels during Laminar and Turbulent Flow Conditions," ASME, Summer Heat Transfer Conference HT2013, Minneapolis, MN. July 14-19 2013. HT2013-17428.

[3] Timchenko, V., Reizes, J., and Leonardi, E., “An Evaluation of Synthetic Jets for Heat Transfer Enhancement in Air Cooled Microchannels", Int. Journal of Numerical Methods for Heat and Fluid Flow, 2007, Vol.17, No.3, pp.263-283.

[4] Timchenko, V, Reizes, JA \& Leonardi, E, 2007, 'The effect of synthetic jet actuators on the convective flow of water in micro-channels.', in Heat and Mass Transfer, presented at Heat and Mass Transfer, Perth, WA, July $26-29,2005$.

[5] Chandratilleke, T., Jagannatha, D., and Narayanaswamy, R. "Performance Analysis of a Synthetic Jet-Microchannel Hybrid Heat Sink for Electronic Cooling," Proc. IEEE $11^{\text {th }}$ Electronics packaging Technology Conference, 2009, pp.630-635.

[6] Travnicek, Z., Dancova, P., Kordik, J., Vit, T., and Pavelka, M., "Heat and Mass Transfer Caused by Laminar Channel Flow Equipped with Synthetic Jet Array," Proc. $14^{\text {th }}$ Int. Heat Transfer Conference HTC14, Washington DC, USA. August 8-13, 2010. Paper Number: IHTC14-22686.

[7] M. Conde Engineering. "Thermophysical Properties of Brines," 2002.

[8] Lee, P. and Garimella, S. "Thermally Developing Flow and Heat Transfer in Rectangular Microchannels of Different Aspect Ratios" International Journal of Heat and Mass Transfer. Vol.49, 2006. Pp.30603067. 